\title{
Magnetic helicity as a probe of magnetic flux-tube dynamics in the solar interior
}

\author{
Takashi Sakurai ${ }^{1}$, Yu Gao ${ }^{2}$, and Kirill Kuzanyan ${ }^{1,2,3}$ \\ ${ }^{1}$ National Astronomical Observatory, Mitaka, Tokyo 181-8588, Japan \\ email: sakurai@solar.mtk.nao.ac.jp \\ ${ }^{2}$ National Astronomical Observatories, Chinese Academy of Sciences, Beijing, China \\ ${ }^{3}$ IZMIRAN, Russian Acacemy of Science, Moscow, Russia
}

\begin{abstract}
Magnetic helicity (volume integral of the product of the magnetic field vector $\boldsymbol{B}$ and the vector potential $\boldsymbol{A}$ ), or its proxy, the current helicity at the surface (surface integral of $\boldsymbol{B} \cdot \boldsymbol{J}$ or $B_{z} J_{z}$ ), is an important quantity which characterizes the helical nature of solar magnetic fields. The current helicity on the Sun shows a tendency, though with large dispersion, that it is positive in the southern hemisphere and negative in the northern hemisphere (the helicity sign rule). However, there are indications that the helicity sign rule may be reversed at activity minimum periods. We will discuss the significance of this property by focusing on the statistical distributions of helicity whether its dispersion follows Gaussian distribution or not.
\end{abstract}

Keywords. Sun:magnetic fields, Sun:interior, magnetohydrodyamics, methods:data analysis

\section{Introduction}

Helical nature of many structures on the Sun has been known for decades (Pevtsov 2002). Some of the helical signatures are morphological and hard to quantify, but the measurements of magnetic vector fields since 1980s have provided quantitative measures of helicity in magnetic fields. Seehafer (1990) collected the values of the force-free parameter $\alpha(\nabla \times \boldsymbol{B}=\alpha \boldsymbol{B})$ from 16 active regions, and found that $\alpha$ tends to be negative (11 regions among 12) in the northern hemisphere and positive (3 regions among 4 ) in the southern hemisphere. This anti-symmetric distribution of $\alpha$ with respect to the latitude is now called "the hemispheric sign rule". The number of samples has increased since then, and for example Longcope et al. (1998) analyzed 203 regions and found that the hemispheric sign rule holds for 110 regions. Therefore, the sign rule is a weak statistical rule and shows large dispersion.

A popular idea to explain the sign rule was proposed by Longcope et al. (1998). Sunspot groups tend to appear tilted from the east-west direction in such a way that the preceding sunspots are closer to the equator (the so-called Joy's law). If a sunspot group is made from an untwisted magnetic flux tube that was created (amplified) at the bottom of the convection zone and floats up to the surface with the influence of the Coriolis force, the tilt of the $\Omega$-shaped rising flux tube as a whole is consistent with Joy's law and leads to positive writhe helicity in the northern hemisphere. Since the total helicity of the tube is assumed to be zero (as it started so), the twist of magnetic field lines in the tube would be negative, to compensate for the positive writhe helicity. This negative twist helicity is supposed to be represented by the negative value of $\alpha$ in the northern hemisphere. However, Pevtsov \& Canfield (1999) found no clear correlation between the sunspot tilt angle and the twist helicity. Pevtsov \& Latshuko (2000) derived large scale distribution of helicity by using $\mathrm{SOHO} / \mathrm{MDI}$ longitudinal magnetograms; the assumption of stationarity made it possible to infer the vector field by utilizing the solar rotation. 
The derived helicity distribution follows the hemispheric sign rule up to high latitudes. This result is also against the interpretation of helicity by attributing it to sunspot tilt angle.

Bao et al. (2000) analyzed many more samples and found that the hemispheric sign rule was satisfied in a sample of 422 regions observed in 1988-1997 (solar cycle 22), but was found violated in a sample of 87 regions observed in 1998-2000 (beginning of cycle 23). Hagino \& Sakurai (2005) derived the value of $\mathrm{d} \alpha / \mathrm{d} \theta$ from 1983 to 2002, which should be negative if the hemispheric sign rule holds, and found that the sign of $\mathrm{d} \alpha / \mathrm{d} \theta$ was positive in the period of sunspot number minimum. However, Pevtsov et al. (2008) did not find such a systematic change in $\mathrm{d} \alpha / \mathrm{d} \theta$ as a function of time. Then, Zhang et al. (2010) analyzed 6205 magnetograms of 984 regions in detail and found that the violation of the sign rule is not simply due to the reversal in $\operatorname{sign}$ of $\mathrm{d} \alpha / \mathrm{d} \theta$, but by more frequent appearance of "wrong helicity" regions near the activity minimum periods. In any case, it is clear that the hemispheric distribution of helicity is not simply due to the action of the Coriolis force which must not change during a solar cycle.

Therefore, whether the hemispheric sign rule is followed or violated may depend on the characteristics of the statistical distribution (the probability distribution function, PDF) of helicity, and particularly how it varies in time. These are the topics to be studied in this paper.

\section{Proxies of helicity}

So far we have used the term "helicity" without definition; for a rigorous formulation, see Berger (1999). From observed vector magnetic fields, we may only infer a proxy to the magnetic helicity. One of such proxies is the current helicity

$$
H_{\mathrm{c}}=\sum_{\text {pixel }} J_{z} B_{z}, \quad \boldsymbol{J} \equiv \nabla \times \boldsymbol{B} .
$$

Here the $z$-axis is normal to the solar surface and is directed toward us. A true current helicity is the volume integral of $\boldsymbol{J} \cdot \boldsymbol{B}$, and equation (2.1) is a surface integral of only a part of $\boldsymbol{J} \cdot \boldsymbol{B}$.

Another proxy is the force-free parameter $\alpha$, which may be estimated by computing linear force-free fields; by changing the value of $\alpha$ one can pick up the value $\alpha_{\text {best }}$ which most successfully reproduces the observed transverse magnetic field vectors.

The value of $\alpha$ may be defined in the photosphere where the force-free approximation is not valid, by carrying out a least-square fit

$$
\sum w\left(J_{z}-\alpha B_{z}\right)^{2}=\min
$$

where $w$ means a weight; $w=1$ gives the usual least-square fit to the model equation $J_{z}=\alpha B_{z}$, namely

$$
w=1: \quad \alpha_{\mathrm{av}}^{(2)}=\frac{\sum J_{z} B_{z}}{\sum B_{z}^{2}}=\text { average of } \frac{J_{z}}{B_{z}} \text { weighted by } B_{z}^{2} .
$$

Similarly we define

$$
w=\frac{1}{\left|B_{z}\right|}: \quad \alpha_{\mathrm{av}}^{(1)}=\frac{\sum J_{z} \operatorname{sign}\left(B_{z}\right)}{\sum\left|B_{z}\right|}=\text { average of } \frac{J_{z}}{B_{z}} \text { weighted by }\left|B_{z}\right|,
$$

and

$$
w=\frac{1}{B_{z}^{2}}: \quad \alpha_{\mathrm{av}}^{(0)}=\frac{\sum \frac{J_{z}}{B_{z}}}{\sum 1}=\text { average of } \frac{J_{z}}{B_{z}}
$$



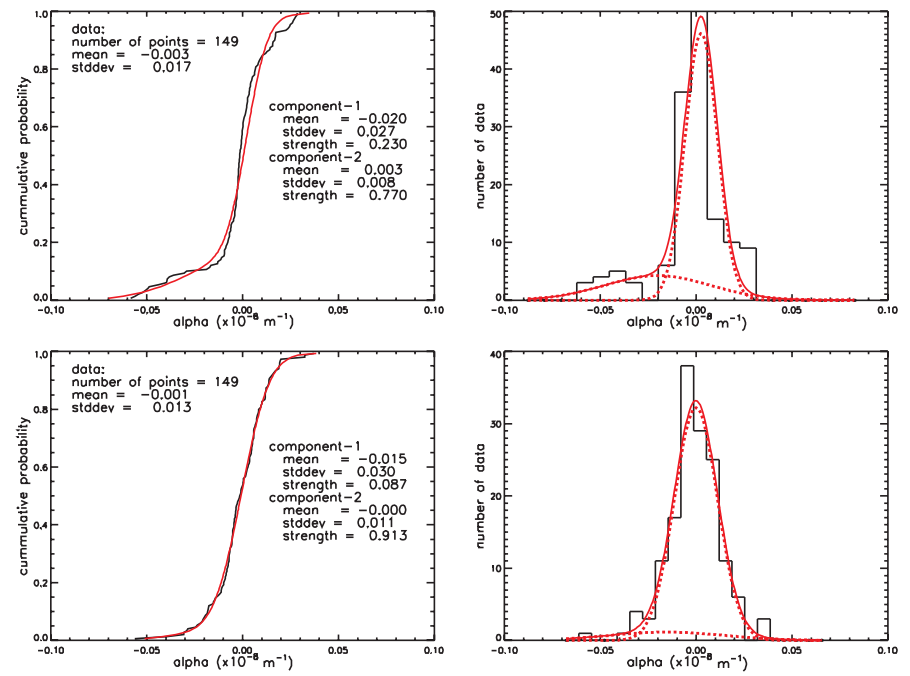

Figure 1. Two examples of the Gaussian decomposition of the observed PDF of parameter $\alpha$. The left column shows the CDF (the smoother curves are the fitted models). The right column shows the derived two Gaussian components (dotted) and their sum (solid) as the PDF, and the histograms. The example in the top row is characterized by a distinct second component, while the example in the bottom row is almost a single Gaussian.

Hagino \& Sakurai (2004) preferred $\alpha_{\mathrm{av}}^{(1)}$, and Hagino \& Sakurai (2005) used the same. The definition of $\alpha_{\mathrm{av}}^{(2)}$ was adopted by Tiwari et al. (2011). For reference, Longcope et al. (1998) used $\alpha_{\mathrm{av}}^{(0)}$, Bao et al. (2000) used $\alpha_{\text {best }}$ and $H_{\mathrm{c}}$, and Pevtsov et al. (2008) used $\alpha_{\text {best }}$. The conclusion of Pevtsov et al. (2008), in which Hagino and Sakurai were coauthors, is against Hagino \& Sakurai (2005), but the former used $\alpha_{\text {best }}$ and the latter adopted $\alpha_{\mathrm{av}}^{(1)}$, and we put more significance in $\alpha_{\mathrm{av}}^{(1)}$ as explained in Hagino \& Sakurai (2004) and $\mathrm{Xu}$ et al. (2012).

\section{Multi-Gaussian decomposition}

The results of Zhang et al. (2010) suggests that the regions that show helicity opposite to the hemispheric rule appear sporadically on top of a more regular background distribution of helicity that conforms to the hemispheric rule. In order to see whether there are two populations in the distribution of helicity, we have tried the following procedure.

If the PDF is made of two Gaussian components, the observed PDF, namely a histogram of distribution in helicity, may look like a superposition of two Gaussian-like histograms. However, the histogram is not a convenient way of performing a fitting to a model because it depends on how the bins are defined. Therefore, we will work on the cumulative distribution function (CDF) which can be uniquely defined by observations. If the observed data are sorted in the ascending order $x_{1}<x_{2} \ldots<x_{n}$, then

$$
\mathrm{CDF}_{\mathrm{obs}}(x)=i / n \text { for } x_{i}<x<x_{i+1}, 0 \leqslant i \leqslant n
$$

with $x_{0}=-\infty$ and $x_{n+1}=+\infty$. We model this CDF by a superposition of two error functions which are specified by their center positions, widths, and amplitudes. The amplitudes must sum up to unity, so that we have five parameters to deal with. Once the best fit $\mathrm{CDF}_{\text {model }}$ is obtained, we can easily derive $\mathrm{PDF}$ which is made of the corresponding Gaussian functions, and we may represent the results by a histogram. 

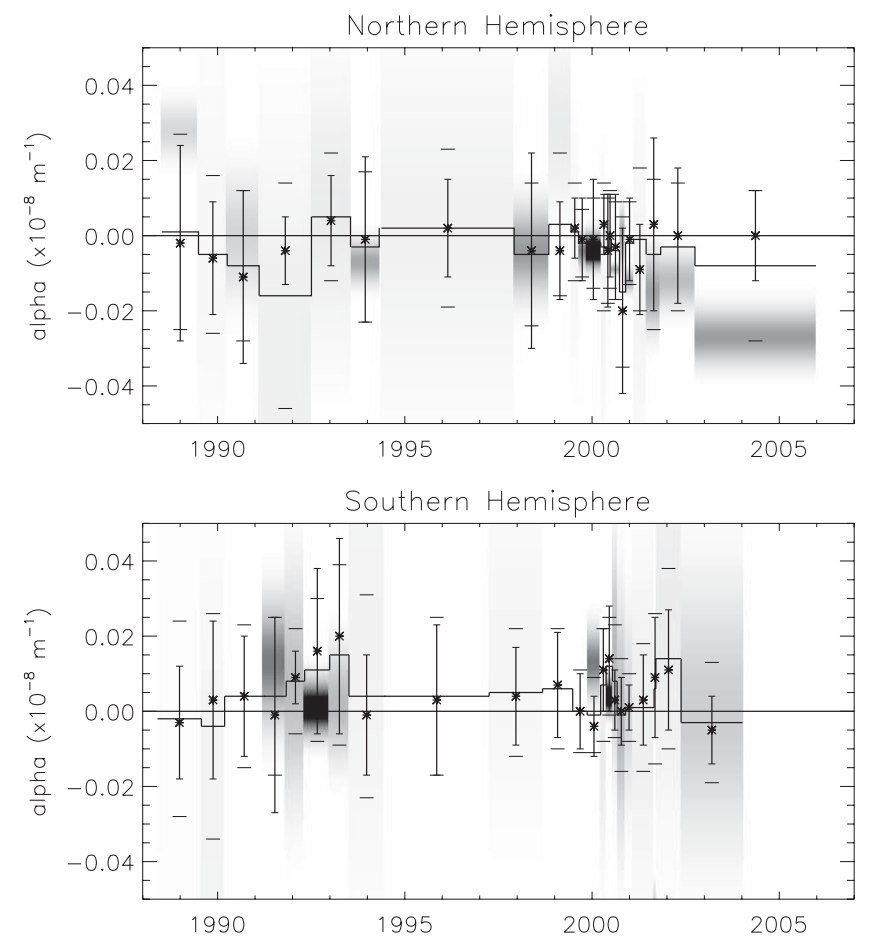

Figure 2. Time variations of the derived Gaussian components of the distributions in $\alpha$ in the northern (top) and southern (bottom) hemispheres. The time is divided into unequal intervals which contain roughly 150 magnetograms each. Step-wise lines and short horizontal bars indicate the mean and standard deviation of the observed data. The asterisks and vertical bars indicate the mean and standard deviation of the main Gaussian component. The gray scales show the sub Gaussian component.

This procedure needs a large number of samples $n$. We tested the fitting procedure on prescribed two-Gaussian models. By varying $n$ and generating random numbers $r_{i}$ $(1 \leqslant i \leqslant n)$ uniformly distributed between 0 and 1 , a simulated data set can be obtained by $x_{i}=\mathrm{CDF}_{\text {model }}^{-1}\left(r_{i}\right)$. We found that generally $n \gtrsim 100$ is required.

Zhang et al. (2010) analyzed the data taken at Huairou Solar Observing Station of National Astronomical Observatories of China from 1988 to 2005. They divided the latitudes into $7^{\circ}$ bins and their time resolution was two years. If we require $n>100$, we have to reduce the latitude resolution. In this study we only distinguish between the northern and southern hemispheres. In time we grouped the data in variable lengths so that each group has more than 150 samples.

Figure 1 shows two examples of fitting to data from the northern hemisphere at different epochs. In some cases (like the one at the bottom), a single Gaussian fit is a reasonable approximation. In other cases (like the one at the top), a distinct second Gaussian component is observed. The next task is to study the statistical properties of these two Gaussian components.

\section{Results}

Figure 2 shows the results based on the data taken at Huairou from 1988 to 2005. The data were analyzed separately for the northern (top panel) and southern (bottom panel) hemispheres. For each hemisphere, the data were divided into unequal intervals in such 


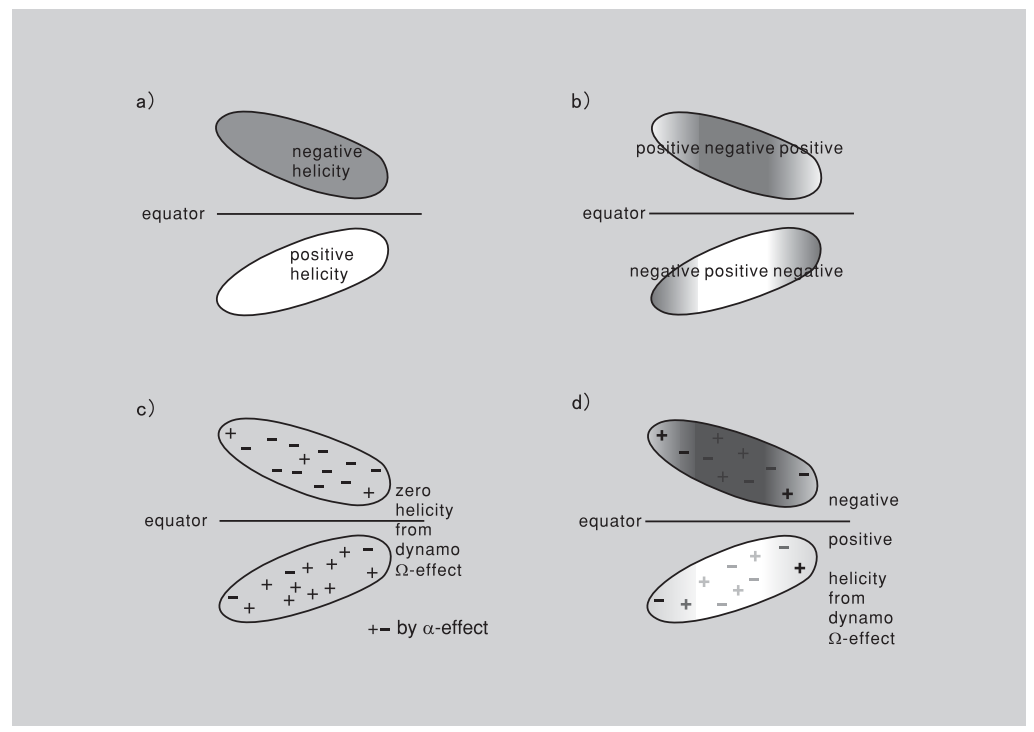

Figure 3. Interpretations of statistical distributions of helicity in the form of a butterfly diagram. (a) The hemispheric sign rule is followed all the time. (b) The sign rule changes its sense near the activity minimum. (c) The sign rule is violated occasionally by the appearance of helicity patches; the background helicity is zero. (d) The background helicity follows the hemispheric sign rule, but when its strength weakens, it is violated occasionally by the appearance of helicity patches.

a way that each interval contains roughly 150 magnetograms. The method described in the previous section has been applied, and parameters of the two Gaussian components were derived. The mean and standard deviation of the observed data ( $\alpha$ in units of $10^{-8} \mathrm{~m}^{-1}$ ) are represented by step-wise lines and short horizontal bars. The mean and standard deviation of the main Gaussian component are represented by asterisks and vertical bars. The sub-component is represented by the gray scales whose intensity is proportional to the probability.

We can notice the following properties.

(1) The main Gaussian component more conforms to the hemispheric sign rule.

(2) The sub-component has on average $20 \%$ in amplitude compared to the main component and shows larger Gaussian width. Its mean is close to zero, but its dispersion is large, which sometimes upsets the hemispheric sign rule.

We had expected that the sub-component may carry helicity which is opposite to the sign rule, but so far we are not able to see such tendency. As a matter of fact, the sub-component of the northern hemisphere follows the hemispheric sign rule (average of Gaussian peak position $=-0.005 \pm 0.014$ ), while the sub-component of the southern hemisphere is slightly against the hemispheric sign rule (average of Gaussian peak position $=-0.001 \pm 0.026)$.

\section{Discussion}

Does the main component represent a primordial twist produced by the dynamo mechanism? What is the origin of sub-component(s)? These are the questions which we want to address.

Figure 3 is our tentative picture in the form of a butterfly diagram. Cases (a) and (b) do not conform to the observed facts but are shown for reference. Case (a) shows that the 
hemispheric sign rule is followed all the time, and the northern (southern) hemisphere shows negative (positive) helicity, respectively. This was our knowledge up to around 2000. Such a persistent sign of helicity implies the effect of the Coriolis force, but we do not believe that the sunspot tilt angle is the origin of helicity, as we described in section 1 .

Case (b) shows that the sign rule reverses its sense near the activity minimum periods, as was advocated by Hagino \& Sakurai (2005). Choudhuri et al. (2004) explained such a reversal by the combination of the dynamo-generated new toroidal field and the old poloidal field from the previous cycle. As Zhang et al. (2010) showed, this is too idealistic a picture, and the violation of the hemispheric rule is not simply the reversal of the rule; it is due to fluctuating appearance of helicity patches of the wrong sign.

In case (c), active regions do not have systematic helicity any time, and helicity is entirely due to fluctuating appearance of helicity patches. It is not clear how the fluctuations can be ordered in such a way that the sign rule is followed in the activity maximum phase. In case (d), active regions have background, persistent helicity which follows the hemispheric rule, but this background helicity weakens in the activity minimum phase. Then the effect of fluctuating helicity patches manifests, and occasionally the hemispheric sign rule is violated. We suppose that the background helicity is due to the dynamo $\Omega$ effect, and fluctuating helicity patches are due to the $\alpha$-effect. The fact that the PDF of $\alpha$ values is mostly represented by two Gaussians may indicate that the $\alpha$-effect is a discrete event. Suppose that a flux tube is generated at the bottom of the convection zone and starts to rise. The flux tube may have helicity from the outset according to the sign rule. If the tube rises as it is, then it shows the hemispheric sign rule. If it encounters with a helical convective cell during its ascent, it acquires additional, fluctuating helicity. Such flux tubes may comprise the second Gaussian component we observe. It is therefore of crucial importance to investigate more in detail the statistical properties of the Gaussian components in the PDF of helicity. It may be that we are beginning to see the $\alpha$-mechanism operating in the solar convection zone.

\section{References}

Ai, G. X. \& Hu, Y. F. 1986, Acta Astron. Sinica, 27, 173

Bao, S. D., Ai, G. X., \& Zhang, H. Q. 2000, JAA, 21, 303

Berger, M. A. 1999, in: M. R. Brown, R. C. Canfield, \& A.A.Pevtsov (eds.), Magnetic Helicity in Space and Laboratory Plasmas, AGU Geophys. Monogr. 111, p. 1

Choudhuri, A. R., Chatterjee, P., \& Nandy, D. 2004, ApJL, 615, L57

Hagino, M. \& Sakurai, T. 2004, PASJ, 56, 831

Hagino, M. \& Sakurai, T. 2005, PASJ, 57, 481

Longcope, D. W., Fisher, G. H., \& Pevtsov, A. A. 1998, ApJ, 507, 417

Pevtsov, A. A. \& Canfield, R. C. 1999, in: M. R. Brown, R. C. Canfield, \& A. A.Pevtsov (eds.), Magnetic Helicity in Space and Laboratory Plasmas, AGU Geophys. Monogr. 111, p. 103

Pevtsov, A. A. \& Latushko, S. M. 2000, ApJ, 528, 999

Pevtsov, A. A. 2002, in: P. C. H. Martens \& D. P. Cauffman (eds.), Multiple Wavelength Observations of Coronal Structures and Dynamnics, COSPAR Colloq. Ser. 13 (Amsterdam: Elsevier Science), p. 125

Pevtsov, A. A., Canfield, R. C., Sakurai, T., \& Hagino, M. 2008, ApJ, 677, 719

Seehafer, N. 1990, Solar Phys., 125, 219

Tiwari, S. K., Venkatakrishnan, P., Gosain, S., \& Joshi, J. 2011, ApJ, 700, 199

Xu, H., Gao, Y., Zhang, H., Sakurai, T., Hagino, M., Sokoloff, D., \& Pevtsov, A. A. 2012, PASJ, 64,54

Zhang, H., Sakurai, T., Pevtsov, A., Gao, Y., Xu, H., Sokoloff, D. D., \& Kuzanyan, K. 2010, MNRAS, 402, L30 